\title{
The Effect of Pulsed Radiofrequency Applied to the Peripheral Nerve in Chronic Constriction Injury Rat Model
}

\author{
Jun-Beom Lee, $\mathrm{MD}^{1}$, Jeong-Hyun Byun, $\mathrm{MD}^{1}$, In-Sung Choi, MD, PhD ${ }^{1}$, \\ Young Kim, $\mathrm{MD}^{2}$, Ji Shin Lee, $\mathrm{MD}, \mathrm{PhD}^{2}$ \\ ${ }^{1}$ Department of Physical and Rehabilitation Medicine, Research Institute of Medical Sciences, \\ Chonnam National University Medical School \& Hospital, Gwangju; \\ ${ }^{2}$ Department of Pathology, Research Institute of Medical Sciences, Chonnam National University Medical School, Gwangju, Korea
}

\begin{abstract}
Objective To investigate the effect of pulsed radiofrequency (PRF) applied proximal to the injured peripheral nerve on the expression of tumor necrosis factor- $\alpha$ (TNF- $\alpha$ ) in a neuropathic pain rat model.

Methods Nineteen male Sprague-Dawley rats were used in the study. All rats underwent chronic constriction injury (CCI) procedure. After 7 days of CCI, withdrawal frequency of affected hind paw to mechanical stimuli and withdrawal latency of affected hind paw to heat stimulus were measured. They were randomly divided into two groups: group A, CCI group ( $n=9)$ and group $B, C C I$ treated with PRF group $(n=10)$. Rats of group B underwent PRF procedure on the sciatic nerve. Withdrawal frequency and withdrawal latency were measured at 12 hours, and 7 days after PRF. Immunohistochemistry and Western blot analysis were performed using a TNF- $\alpha$ antibody.

Results Before PRF, withdrawal frequency and withdrawal latency were not different in both groups. After PRF, withdrawal frequency decreased and withdrawal latency prolonged over time in group B. There was significant interaction between time and group for each withdrawal frequency and withdrawal latency. Group B showed decreased TNF- $\alpha$ immunoreactivity of the spinal cord and sciatic nerve at 7 days.

Conclusion PRF applied proximal to the peripheral nerve injury is potentially helpful for the reduction of neuropathic pain by neuromodulation of inflammatory markers.
\end{abstract}

Keywords Neuralgia, Pulsed radiofrequency treatment, Peripheral nerves, Tumor necrosis factor-alpha

Received April 15, 2015; Accepted May 29, 2015

Corresponding author: In-Sung Choi

Department of Physical and Rehabilitation Medicine, Chonnam National University Medical School \& Hospital, 42 Jebong-ro, Dong-gu, Gwangju 61469, Korea

Tel: +82-62-220-5606, Fax: +82-62-228-5975, E-mail: drchoiis@naver.com

@ This is an open-access article distributed under the terms of the Creative Commons Attribution Non-Commercial License (http://creativecommons. org/licenses/by-nc/4.0) which permits unrestricted noncommercial use, distribution, and reproduction in any medium, provided the original work is properly cited.

Copyright $\odot 2015$ by Korean Academy of Rehabilitation Medicine

\section{INTRODUCTION}

Neuropathic pain is defined as pain caused by a lesion or disease of the somatosensory nervous system [1]. Neuropathic pain is one of the chronic intractable pain syndromes. If pain is not controlled at an early stage, it may progress to refractory pain and limit activities of daily living independence [2,3]. Existing pharmacologic treatments for neuropathic pain are limited, with partial relief of pain in $40 \%-60 \%$ of patients [2]. Therefore, there is 
growing attention to non-pharmacologic managements for neuropathic pain.

Radiofrequency (RF) has long been used for the treatment of neuropathic pain $[4,5]$. RF treatment is classified as conventional RF (CRF) and pulsed RF (PRF) [6]. CRF uses frictional heat generated from the catheter needle. A wide range of thermal damage due to RF stimulation can cause nerve damage. Unlike CRF, PRF has the advantages of safe application and fewer side effects, because application sites maintain the temperature below $42^{\circ} \mathrm{C}$ $[7,8]$. Biological effects of PRF differ from CRF, but action mechanism of PRF is still unknown. One of the current hypotheses is neuromodulation through gene expression change [9]. Tumor necrosis factor- $\alpha$ (TNF- $\alpha$ ) as a pain modulating factor, is involved in the development of neuropathic pain after peripheral nerve injury [10-12]. TNF- $\alpha$ protein is increased in injured peripheral nerve and dorsal root ganglion (DRG) and increased immunoreactivity is detected in glial cell and nerve cell body after chronic constriction injury (CCI)-induced neuropathic pain [13]. Our hypothesis was that PRF applied proximal to the injured peripheral nerve would decrease neuropathic pain symptoms, which affect the level of TNF- $\alpha$ in the peripheral nerve and spinal cord.

While RF to DRG requires fluoroscopic guidance, approach to the peripheral nerve is relatively easy with recent prevalence of musculoskeletal ultrasonography. If PRF applied to the peripheral nerve is effective for pain control, it could be developed as an easy and comfortable therapeutic tool for neuropathic pain patients. Recent human studies showed positive results [14,15]. Animal studies are helpful in understanding the therapeutic effects and the action mechanisms of various treatments, however, animal studies on the application of PRF to the injured peripheral nerve are rare. We aimed to investigate the effect of PRF applied to sciatic nerve on neuropathic pain in CCI rat model.

\section{MATERIALS AND METHODS}

\section{Subjects}

Nineteen male Sprague-Dawley rats, weighing 250-300 grams, aged 10 weeks old, were used in the study. The rats were housed at a temperature of $22^{\circ} \mathrm{C} \pm 2^{\circ} \mathrm{C}$ and a humidity of $55 \%$ with a 12 -hour light/dark cycle (light on 8 AM to 8 PM) with free access to food and water ad libi- tum.

All rats underwent CCI procedure as described by Bennett and Xie [16]. Withdrawal frequency and withdrawal latency were measured to evaluate whether neuropathic pain was induced on the 7th day after surgery. Rats were randomly divided into two groups according to the application of PRF: group A, CCI group ( $\mathrm{n}=9)$ and group $\mathrm{B}$, CCI treated with PRF group $(n=10)$. Group B was treated with PRF. All animal experiments were conducted in accordance with the guidelines of the Chonnam National University Animal Care and Use Committee.

\section{Induction of neuropathic pain}

Unilateral CCI of right sciatic nerve was induced in all rats [16]. Rats were anesthetized with tiletamine $\mathrm{HCl} /$ zolazepam HCl (Zoletil; Virbac, Carros, France) $50 \mathrm{mg} /$ kg and xylazine (Rompun; Bayer Korea, Seoul, Korea) 7 $\mathrm{mg} / \mathrm{kg}$ intraperitoneally. In brief, we removed the hair of right gluteal region and disinfected the skin with povidone iodine. The skin was opened over a length of $2 \mathrm{~cm}$ in the proximal half of the line between trochanter and knee joint. The biceps femoris muscle was longitudinally separated and the sciatic nerve was exposed under the muscle. Proximal to the trifurcation of sciatic nerve (tibial, sural and common peroneal nerves), 4 loose ligatures of 4-0 chromic catgut at an interval of $1 \mathrm{~mm}$ were placed around the sciatic nerve. After the induction of CCI, the skin and muscle fascia were sutured. For the prevention of infection, gentamicin sulfate $(5 \mathrm{mg} / \mathrm{kg}$ ) was administered into the gluteal muscle for 3 days. All surgical procedures were performed by a researcher.

\section{Pulsed radiofrequency}

All rats of group B were anesthetized intraperitoneally and the sciatic nerves with CCI were exposed again. The rats underwent PRF procedure on the sciatic nerve using a $10-\mathrm{cm}$ long electrode with a diameter of $0.55 \mathrm{~mm}$ and exposure length of $1 \mathrm{~mm}$ powered by an RF generator (PMG-230; Baylis Medical Company Inc., Montreal, Canada). Probe for PRF stimulation was fixed to a stereotactic frame and 1-2 mm of the distance between probe and the sciatic nerve was placed to avoid direct mechanical stimulation [17]. The lesion generator was set to deliver $\mathrm{RF}$ proximal $1 \mathrm{~mm}$ to the ligatures in the exposed sciatic nerve [18] at 2 bursts per second of $20 \mathrm{~ms}$ duration each with the automatic temperature control activated and set 
to $42^{\circ} \mathrm{C}$ for 120 seconds. PRF was applied twice at an interval of 1 minute [19].

\section{Evaluation of neuropathic pain}

Withdrawal frequency and withdrawal latency were measured before and at 12 hours and 7 days after PRF. For the evaluation of mechanical allodynia, withdrawal frequency of the hind paw was measured by determining paw withdrawal after probing the paw with a series of calibrated monofilaments, Touch Test Sensory Evaluator (North Coast Medical Inc., Gilroy, CA, USA) [20]. All rats were placed on a metal mesh and the plantar surface of the operated hind paw was touched with Von Frey monofilament with a bending force of $5.07 \mathrm{mN}$. The occurrence of withdrawal in response to innocuous mechanical stimuli was measured at a stimulation rate of $0.2 \mathrm{~Hz}$ in 20 trials.

For the evaluation of thermal hyperalgesia, withdrawal latency to the heat stimulus was measured by paw withdrawal from a focused beam of radiant heat, Plantar test (Ugo Basile, Varese, Italy) onto the surface of the hind paw [21]. All rats were placed in a plastic box with a 3 -mm thick glass floor and allowed to acclimate to their environment before testing. A mobile radiant infrared source under the transparent glass floor was applied to the plantar surface of right hind paw. Withdrawal latency was defined as the time from the onset of the heat stimulus to the withdrawal of the hind paw. The intensity of the heat stimulus was 55 . A cut-off time of 30 seconds was set to avoid possible tissue damage. Each paw was tested twice with at least 5 minutes interval between consecutive tests. The mean value of the 2 trials was used for data analysis.

\section{Immunohistochemistry}

The sampled rats of each group were sacrificed at 12 hours and 7 days after PRF. Rats were deeply anaesthetized with $2.5 \%$ pentotal sodium, and the heart was then exposed. Each rat was transcardially perfused with 100 $\mathrm{mL}$ saline, followed by phosphate buffer $(0.1 \mathrm{~mol} / \mathrm{L} \mathrm{PB}$, $\mathrm{pH}$ 7.4) of $4 \%$ paraformaldehyde $400 \mathrm{~mL}$. The sciatic nerves were removed from $5 \mathrm{~mm}$ proximal to the lesion to $3 \mathrm{~mm}$ distal to the lesion and the spinal cords $(2 \mathrm{~cm}$ long) were removed from lumbar enlargement, fixed in $10 \%$ formalin solution for 48 hours and embedded in paraffin wax. Paraffin embedding tissues of sciatic nerve and spinal cord were cut axially at $5 \mu \mathrm{m}$ thickness. After being posted on the slide, tissues were treated with xylene and deparaffinized. The sections were dehydrated in graded alcohol. After sections were rinsed in phosphate-buffered saline (PBS, $\mathrm{pH}$ 7.4), nerve sections were then incubated with $3 \%$ hydrogen peroxide for 30 minutes. Sections were incubated with TNF- $\alpha$ protein antibody as primary antibody diluted 1:500. Incubations with the primary antibody were conducted overnight at $4^{\circ} \mathrm{C}$, sections were then washed thrice with PBS and incubated with quick anti-rabbit/anti-mouse immunoglobulin for 30 minutes. Sections were subsequently washed thrice with PBS and incubated with streptavidin peroxidase for 10 minutes. The same sections were washed and reacted with 3,3' -diaminobenzidine (DAB; DAKO Corp., Carpinteria, CA, USA) for 10 minutes at room temperature and counterstained with Mayer's hematoxylin. TNF- $\alpha$ expression was evaluated under a light microscope (Nikon, Tokyo, Japan) at a magnification of 200 .

\section{Western blotting}

Rats were rapidly decapitated under intraperitoneal anesthesia. Sciatic nerves and spinal cords were removed and immediately placed in liquid nitrogen and stored at $-70^{\circ} \mathrm{C}$ until use. Rapidly frozen sections were then washed with PBS and kept until manually homogenized in liquid nitrogen to powder form; tissue homogenates were then placed in a 1.5 -mL tube. PRO-PREP Protein Purification Kit (iNtRON Biotechnology, Seongnam, Korea) extraction buffer $600 \mu \mathrm{L}$ solution was added and dispersed. Samples were agitated and centrifuged for 30 minutes at 13,000 rpm to separate supernatant $200 \mu \mathrm{L}$ at $4^{\circ} \mathrm{C}$ and transferred to a new tube. Protein was diluted and quantified with reading by ELISA using Pierce BCA Protein Assay Kit (Thermo Fisher Scientific Inc., Waltham, MA, USA). After mixing the sample buffer, NuPAGE (Invitrogen, Carlsbad, CA, USA), protein denaturation was induced by heating for 5 minutes at $38^{\circ} \mathrm{C}$. Denatured proteins were separated by electrophoresis on $12 \%$ acrylamide gel and transferred to nitrocellulose membranes. The membranes were blocked in 5\% skim milk/TBS-T for 1 hour at room temperature and TNF- $\alpha$ antibody 1:1,000, in 3\% skim milk/ TBS-T (Novus Biologicals, Littleton, CO, USA) was incubated overnight at $4^{\circ} \mathrm{C}$. The membranes were washed in Tris-bufferd saline with $0.1 \%$ Tween-20 (TBS-T). The blots were incubated in membrane for 1 hour at room 
temperature with goat anti-rabbit IgG-HRP 1:5,000, in 1\% skim milk/TBS-T (Santa Cruz Biotechnology, Santa Cruz, CA, USA). After washing with Tris-bufferd saline (TBS-T), immunoreactive protein bands were visualized using the enhanced chemiluminescence, Immobilon Western Chemiluminescent Kit (Merck Millipore, Billerica, MA, USA) and LAS-3000 Imaging System (Fujifilm, Tokyo, Japan). After the images of bands were scanned in grayscale at a resolution of $600 \mathrm{dpi}$, the bands were quantified using ImageJ (National Institute of Health, Bethesda, MD, USA).

\section{Statistical analyses}

Statistical analyses were performed using SPSS ver. 21 statistical software (IBM SPSS, Armonk, NY, USA). The results were expressed as mean \pm standard deviation. Repeated measures analysis of variance (RM-ANOVA) was used to study the differences between groups and the changes with time in behavioral tests. Post hoc testing consisted of multiple comparisons using the Bonferroni method. Mann-Whitney U-test was used to compare the TNF- $\alpha$ immunoreactivity between both groups. A difference was accepted as significant if $\mathrm{p}<0.05$.

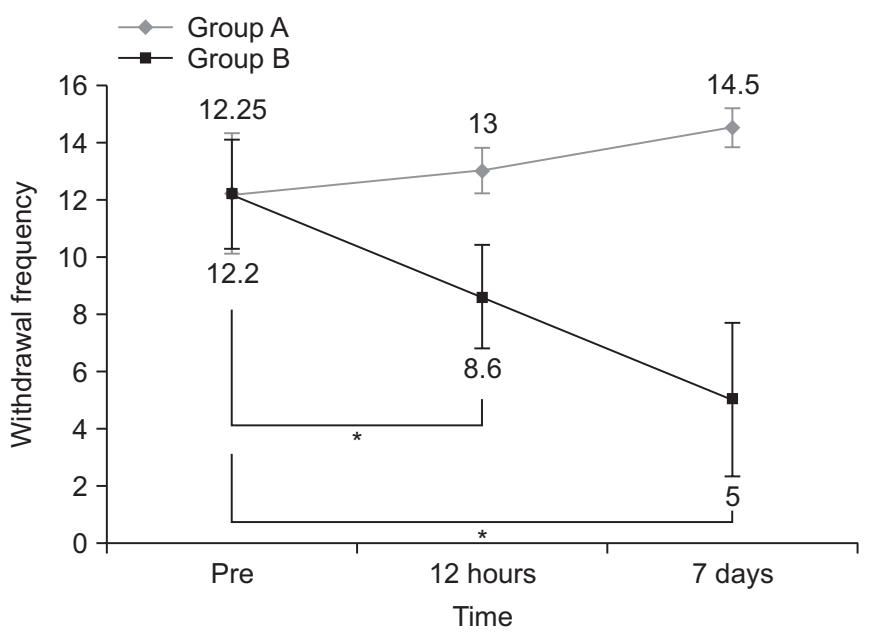

Fig. 1. The effect of pulsed radiofrequency (PRF) on mechanical allodynia. Withdrawal frequency decreases over time in group B. There is significant interaction between time and group for withdrawal frequency. There are significant differences in withdrawal frequency between before and 12 hours after PRF, and between before and 7 days after PRF. ${ }^{*} \mathrm{p}<0.05$.

\section{RESULTS}

Before PRF, there were no significant differences between group A and group B in withdrawal frequency ( $p>0.05)$ and withdrawal latency $(p>0.05)$, respectively. Withdrawal frequency decreased over time in group B. There was significant within-subjects effect $(p=0.002)$ and between-subjects effect $(\mathrm{p}=0.004)$. In pairwise comparisons, there were significant difference of withdrawal frequency between before and 12 hours after PRF ( $p=0.042)$ and between before and 7 days after PRF ( $p=0.026)$. There was significant interaction between time and group for withdrawal frequency $(\mathrm{p}<0.001)$ (Fig. 1). Withdrawal latency increased over time in group $B$. There was significant within-subjects effect $(p<0.001)$ and betweensubjects effect $(p<0.001)$. In pairwise comparisons, there were significant difference of withdrawal latency between before and at 7 days after PRF ( $\mathrm{p}=0.001)$. There was significant interaction between time and group for withdrawal latency $(\mathrm{p}<0.001)$ (Fig. 2).

TNF- $\alpha$ immunoreactivities were observed in the sciatic nerves and the neurons of spinal cord dorsal horn in both groups. Few TNF- $\alpha$-positive stained cells were detected in the spinal cord at 7 days after PRF stimulation (Fig. 3). The levels of TNF- $\alpha$ protein expression were not significantly different between both groups at 12 hours after PRF. However, the protein expression of TNF- $\alpha$ was down-regulated in the spinal cord $(p=0.04)$ and sciatic

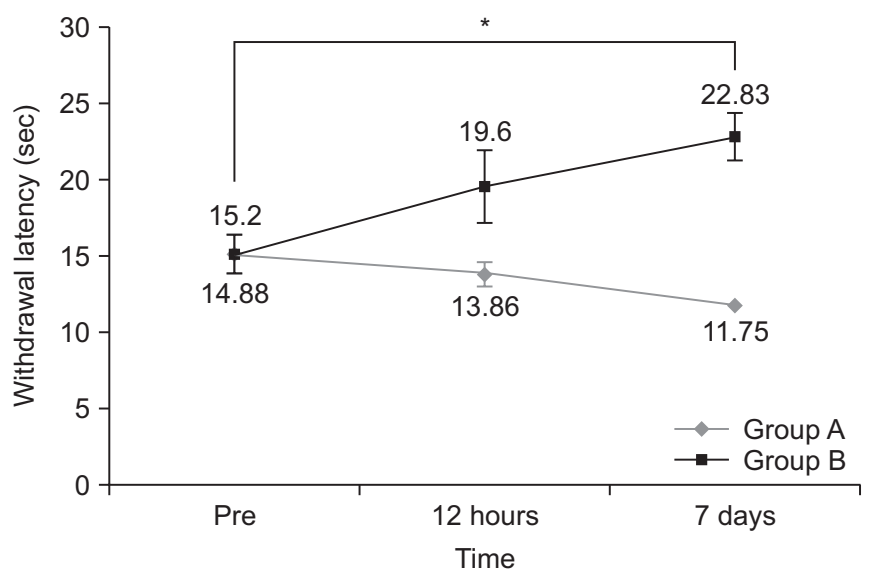

Fig. 2. The effect of pulsed radiofrequency (PRF) on thermal hyperalgesia. Withdrawal latency increases over time in group B. There is significant interaction between time and group for withdrawal latency. There are significant differences in withdrawal latency between before and 7 days after PRF. *p<0.05. 


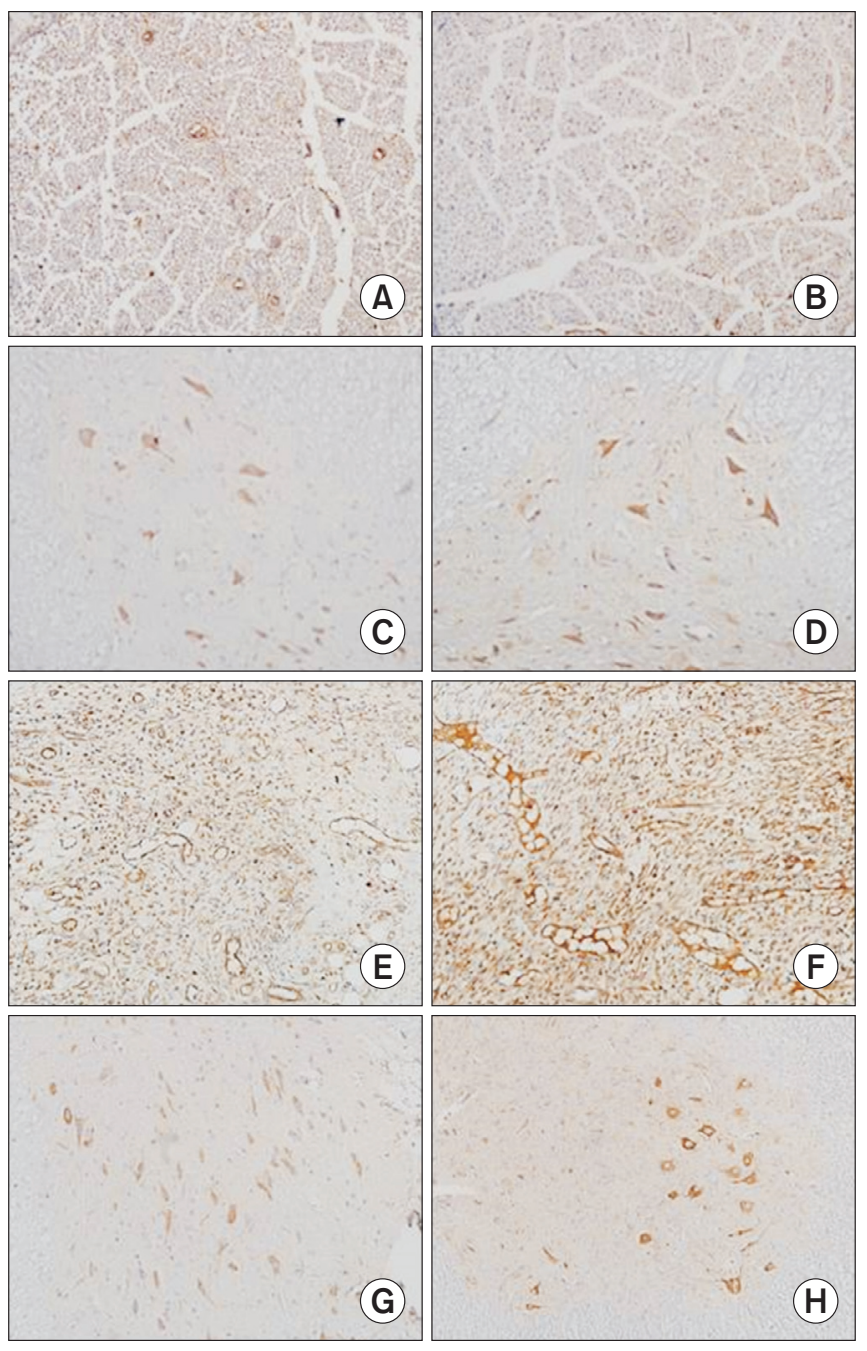

Fig. 3. Tumor necrosis factor- $\alpha$ (TNF- $\alpha$ ) immunoreactivities in the sciatic nerves and the neurons of spinal cord dorsal horn $(\times 200)$. Few TNF- $\alpha$-positive stained cells are detected in the spinal cord at 7 days after pulsed radiofrequency stimulation. Left column is group $A$; right column is group B. (A, B) Sciatic nerve at 12 hours. (C, D) Dorsal horn of spinal cord at 12 hours. (E, F) Sciatic nerve at 7 days. $(G, H)$ Dorsal horn of spinal cord at 7 days.

nerve $(\mathrm{p}=0.03)$ of group $B$ than group A at 7 days after PRF (Fig. 4).

\section{DISCUSSION}

Neuropathic pain can result from peripheral nerve injuries, which trigger maladaptive alterations in the nervous system leading to peripheral and central sensitization that underlie transition to chronic pain [22]. Neuropathic pain can lead to a number of symptoms such as sponta-
(A)
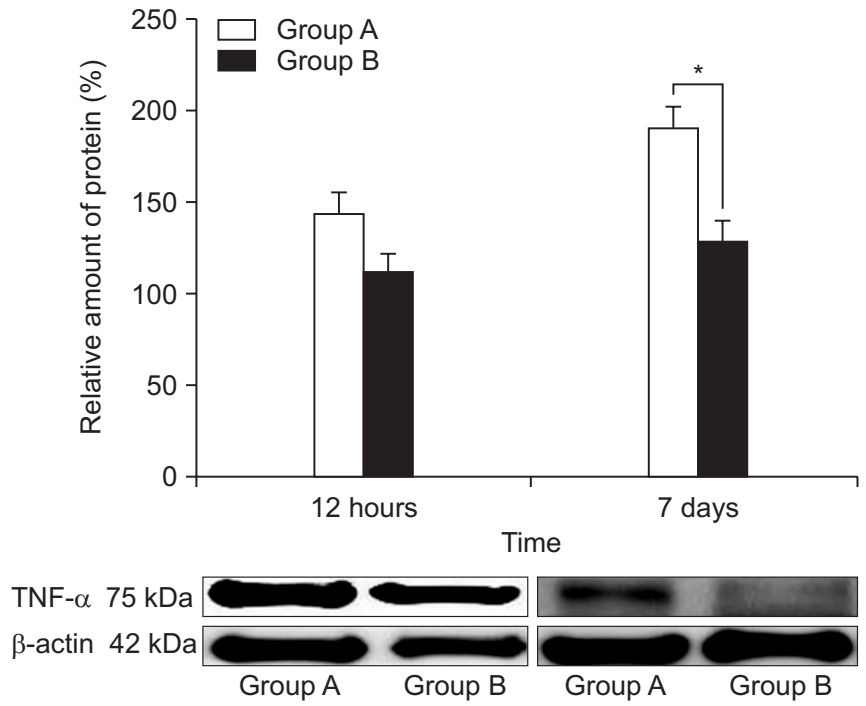

(B)

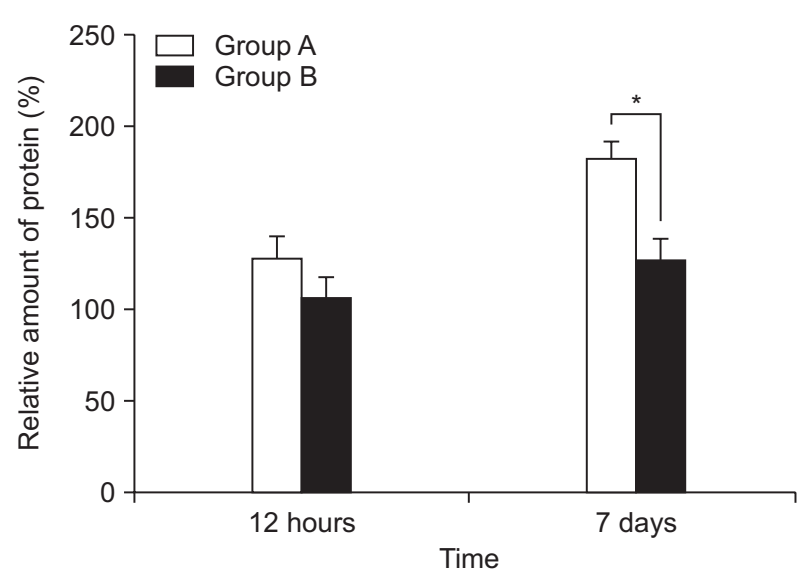

TNF- $\alpha$

$75 \mathrm{kDa}$

$\beta$-actin $42 \mathrm{kDa}$

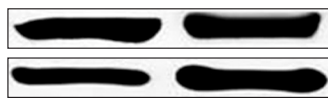

Group A

Group B

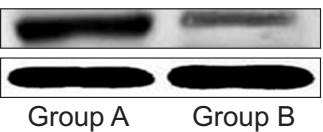

Fig. 4. The levels of tumor necrosis factor- $\alpha$ (TNF- $\alpha$ ) protein expression are not significantly different between both groups at 12 hours after pulsed radiofrequency (PRF). However, the protein expression of TNF- $\alpha$ is down-regulated in the sciatic nerve (A) and spinal cord (B) of group B than group A at 7 days after PRF. ${ }^{*} \mathrm{p}<0.05$.

neous pain, allodynia and hyperalgesia [1]. CCI model is a commonly used rat model for neuropathic pain [16]. In CCI model, thermal hyperalgesia occurs with loose ligation around the sciatic nerve without actual mechanical damage [23]. Thus, the change in inflammatory microenvironment rather than nerve injury per se is important for the development of neuropathic pain $[24,25]$. TNF- $\alpha$ is a proinflammatory cytokine produced by peripheral nerve 
glia [26]. Local sciatic injection of TNF- $\alpha$ caused significant thermal hyperalgesia and mechanical allodynia for 3 days post-injection in association with nerve edema, splitting of myelin lamellae with vacuolization, Schwann cell injury, fibroblast and macrophage activation, and phagocytosis of lipid debris. TNF- $\alpha$ is implicated in neuropathic pain pathologies [27]. TNF- $\alpha$ is associated with the development of neuropathic pain in various animal models. CCI led to the significant development of neuropathic pain symptoms at 14 days after surgery [28]. CCI of the sciatic nerve induces TNF- $\alpha$ expression in the sciatic nerve [29]. In addition, expression of TNF- $\alpha$ and generation of tactile allodynia/hyperalgesia are correlated [30]. TNF- $\alpha$ appears early in the cytokine cascade. TNF- $\alpha$ protein expression displayed 2 distinct peaks ( 6 hours and 5 days) in its temporal profile after CCI [31]. TNF- $\alpha$ produced at the nerve injury site is axonally transported to DRG neurons and the dorsal horn of spinal cord after CCI of the rat sciatic nerve [31]. In this study, CCI group without PRF treatments displayed significant neuropathic pain symptoms and elevated TNF- $\alpha$ level in the sciatic nerve and the spinal cord. Elevated levels of TNF- $\alpha$ in the sciatic nerve at 14 days after CCI are consistent with other studies [28]. Increased levels of TNF- $\alpha$ in sciatic nerve and spinal cord in CCI rats concomitant with the development of mechanical and thermal hyperalgesia clearly indicate a role for cytokines in neuropathic pain [32].

$\mathrm{RF}$ treatment is increasingly used in the management of neuropathic pain. CRF is a neuro-destructive procedure using heat. It slows nerve regeneration by denaturation of protein, which occurs at $>60^{\circ} \mathrm{C}$ and nonspecific destruction of nervous continuity, leading to long-duration treatment effect than other nerve blocks [33]. PRF is known to have analgesic effect by mechanisms other than heat transfer [7]. Exposure of DRG to PRF induces c-Fos expression in lamina I and II of the dorsal horn, unlike CRF exposure, suggestive of an early cellular response to the electric/dielectric forces generated within the DRG rather than a thermal effect [9]. Because PRF does not produce tissue injury around catheter needle by excessive heat, there is no danger of deafferentation pain [34]. Application of PRF to sciatic nerve and DRG causes transient changes that do not progress to overt axonal injury [19]. PRF resulted in subclinical changes including endoneurial edema caused by alterations in the function of the blood brain barrier, fibroblast activation, and col- lagen deposition. Tissue returned to normal conditions by 7 days in sciatic nerve and 21 days in the DRG. PRF treatment does not rely on thermal injury to achieve its clinical effect [19]. PRF is known to be nondestructive, but, it may produce heat spikes exceeding $45^{\circ} \mathrm{C}-50^{\circ} \mathrm{C}$, causing destructive effects on neurons [35]. In this study, although we could not completely exclude thermal injury by PRF, we maintained the temperature of needle tip at $42^{\circ} \mathrm{C}$ to minimize the probable thermal injury. The parameters used for PRF in this study were the same as other reports [19].

PRF is considered as a nondestructive and reversible neuromodulation intervention [36]. Neuromodulation is a normal property of the nervous system that regulates or modifies electrical impulses flowing through neural tissues. Neuromodulation of nociceptive information takes place in spinal synapses [36]. PRF may relieve neuropathic pain by inducing long-term depression of synaptic transmission in the spinal cord [37]. Tanaka et al. [34] reported that decreased mechanical allodynia was observed at an early stage within 1 week of applying PRF. Aksu et al. [38] reported that PRF stimulation performed on 5-6 lumbar dorsal root improves hyperalgesia in sciatic neuropathy induced rabbit. However, animal study on effect of PRF conducted in peripheral neuropathy area is still lacking. In this study, improvement of mechanical allodynia and thermal hyperalgesia was observed after PRF stimulation to the injured sciatic nerve area. Both mechanical allodynia and thermal hyperalgesia was improved on 7 days after applying PRF. The TNF- $\alpha$ immunoreactivity was decreased in spinal cord and sciatic nerve on 7 days after PRF indicating that PRF stimulation affects modulation of inflammatory response in the injured area. Following peripheral nerve injury, immunoregulatory factors such as TNF- $\alpha$ are induced at the site of injury and undergo retrograde transport to the DRG and anterograde transport from DRG to the site of injury. In a sciatic neuropathy rat model, down-regulation of TNF- $\alpha$ in both the sciatic nerve and DRG indicates that PRF can reduce the expression of pro-inflammatory cytokines in adjacent tissues either directly or indirectly [39]. As TNF- $\alpha$ is one of the major players in the development and maintenance of neuropathic pain [12], the decreased expression of TNF- $\alpha$, and modulation of its target cytokines, indicates that PRF potentially alleviates neuropathic pain states by attenuating neuroinflammation at 
the molecular level [12].

While PRF applied to DRG induces changes in the neurons of dorsal horn, percutaneous application of PRF can be targeted to neural tissues and nociceptors in the periphery [40]. Application of PRF to a patient with meralgia paresthetica under ultrasonographic guidance showed excellent pain relief [14]. A randomized controlled trial demonstrated that the application of PRF to suprascapular nerve under ultrasonographic guidance combined with physical therapy results in pain relief, reduced disability, and improvement of passive range of shoulder motion [15]. Although recent application of PRF to peripheral nerve for easy access control of neuropathic pain is now increasing, it has not been extensively studied. This prompted us to select PRF application to the peripheral nerve.

There were several limitations to this study. We did not use percutaneous approach using ultrasonography for PRF treatments. In human studies, it is obviously less invasive to use percutaneous approach to the peripheral nerve causing neuropathic pain under ultrasonographic guidance. As this was an animal study, we exposed the sciatic nerve to secure clear visibility. The changes of spinal glial cells and glial activation signaling pathway involved in the transmission of neuropathic pain after peripheral nerve injury were not studied. Activated glial cells after nerve injury release various pain-modulating substances, however, studies on other inflammatory markers were insufficient.

In conclusion, these results suggested that PRF stimulation applied proximal to the injured peripheral nerve alleviated neuropathic pain by CCI. Furthermore, the reduction of neuropathic pain after PRF treatments may be associated with neuromodulation effects through the inhibition of the expression or anterograde transportation of TNF- $\alpha$.

\section{CONFLICT OF INTEREST}

No potential conflict of interest relevant to this article was reported.

\section{ACKNOWLEDGMENTS}

This study was financially supported by Chonnam National University in 2011.

\section{REFERENCES}

1. The IASP Taxonomy Working Group. Classification of chronic pain. 2nd rev. ed. Seattle: IASP Press; 2012.

2. Dworkin RH, O'Connor AB, Backonja M, Farrar JT, Finnerup NB, Jensen TS, et al. Pharmacologic management of neuropathic pain: evidence-based recommendations. Pain 2007;132:237-51.

3. Jensen MP, Chodroff MJ, Dworkin RH. The impact of neuropathic pain on health-related quality of life: review and implications. Neurology 2007;68:1178-82.

4. van Wijk RM, Geurts JW, Wynne HJ. Long-lasting analgesic effect of radiofrequency treatment of the lumbosacral dorsal root ganglion. J Neurosurg 2001;94(2 Suppl):227-31.

5. Pagura JR. Percutaneous radiofrequency spinal rhizotomy. Appl Neurophysiol 1983;46:138-46.

6. Vatansever D, Tekin I, Tuglu I, Erbuyun K, Ok G. A comparison of the neuroablative effects of conventional and pulsed radiofrequency techniques. Clin J Pain 2008;24:717-24.

7. Heavner JE, Boswell MV, Racz GB. A comparison of pulsed radiofrequency and continuous radiofrequency on thermocoagulation of egg white in vitro. Pain Physician 2006;9:135-7.

8. Choi GS, Ahn SH, Cho YW, Lee DK. Short-term effects of pulsed radiofrequency on chronic refractory cervical radicular pain. Ann Rehabil Med 2011;35:826-32.

9. Higuchi Y, Nashold BS Jr, Sluijter M, Cosman E, Pearlstein $\mathrm{RD}$. Exposure of the dorsal root ganglion in rats to pulsed radiofrequency currents activates dorsal horn lamina I and II neurons. Neurosurgery 2002;50: 850-6.

10. Xu JT, Xin WJ, Zang Y, Wu CY, Liu XG. The role of tumor necrosis factor-alpha in the neuropathic pain induced by Lumbar 5 ventral root transection in rat. Pain 2006; 123:306-21.

11. Sommer C, Schmidt C, George A. Hyperalgesia in experimental neuropathy is dependent on the TNF receptor 1. Exp Neurol 1998;151:138-42.

12. Leung L, Cahill CM. TNF-alpha and neuropathic pain: a review. J Neuroinflammation 2010;7:27.

13. Jancalek R, Dubovt P, Svizenska I, Klusakova I. Bilateral changes of TNF-alpha and IL-10 protein in the lumbar and cervical dorsal root ganglia following a unilateral chronic constriction injury of the sciatic 
nerve. J Neuroinflammation 2010;7:11.

14. Fowler IM, Tucker AA, Mendez RJ. Treatment of meralgia paresthetica with ultrasound-guided pulsed radiofrequency ablation of the lateral femoral cutaneous nerve. Pain Pract 2012;12:394-8.

15. Wu YT, Ho CW, Chen YL, Li TY, Lee KC, Chen LC. Ultrasound-guided pulsed radiofrequency stimulation of the suprascapular nerve for adhesive capsulitis: a prospective, randomized, controlled trial. Anesth Analg 2014;119:686-92.

16. Bennett GJ, Xie YK. A peripheral mononeuropathy in rat that produces disorders of pain sensation like those seen in man. Pain 1988;33:87-107.

17. Ko HY, Shin YB, Lee SH, Moohn HN, Kwon DR, Ahn $\mathrm{YH}$. Effect of radiofrequency lesioning on peripheral nerve conductivity in relation to distance between lesioning electrode and target tissue in rats. J Korean Acad Rehabil Med 2004;28:449-53.

18. Park CH, Lee YW, Kim YC, Moon JH, Choi JB. Treatment experience of pulsed radiofrequency under ultrasound guided to the trapezius muscle at myofascial pain syndrome: a case report. Korean J Pain 2012;25:524.

19. Podhajsky RJ, Sekiguchi Y, Kikuchi S, Myers RR. The histologic effects of pulsed and continuous radiofrequency lesions at 42 degrees $C$ to rat dorsal root ganglion and sciatic nerve. Spine (Phila Pa 1976) 2005;30: 1008-13.

20. Chaplan SR, Bach FW, Pogrel JW, Chung JM, Yaksh TL. Quantitative assessment of tactile allodynia in the rat paw. J Neurosci Methods 1994;53:55-63.

21. Hargreaves K, Dubner R, Brown F, Flores C, Joris J. A new and sensitive method for measuring thermal nociception in cutaneous hyperalgesia. Pain 1988;32:7788.

22. Costigan M, Scholz J, Woolf CJ. Neuropathic pain: a maladaptive response of the nervous system to damage. Annu Rev Neurosci 2009;32:1-32.

23. Maves TJ, Pechman PS, Gebhart GF, Meller ST. Possible chemical contribution from chromic gut sutures produces disorders of pain sensation like those seen in man. Pain 1993;54:57-69.

24. Sommer C, Galbraith JA, Heckman HM, Myers RR. Pathology of experimental compression neuropathy producing hyperesthesia. J Neuropathol Exp Neurol 1993;52:223-33.
25. Clatworthy AL, Illich PA, Castro GA, Walters ET. Role of peri-axonal inflammation in the development of thermal hyperalgesia and guarding behavior in a rat model of neuropathic pain. Neurosci Lett 1995;184:58.

26. Wagner R, Myers RR. Schwann cells produce tumor necrosis factor alpha: expression in injured and noninjured nerves. Neuroscience 1996;73:625-9.

27. Wagner R, Myers RR. Endoneurial injection of TNFalpha produces neuropathic pain behaviors. Neuroreport 1996;7:2897-901.

28. Kukkar A, Singh N, Jaggi AS. Neuropathic pain-attenuating potential of aliskiren in chronic constriction injury model in rats. J Renin Angiotensin Aldosterone Syst 2013;14:116-23.

29. Wagner R, Janjigian M, Myers RR. Anti-inflammatory interleukin-10 therapy in CCI neuropathy decreases thermal hyperalgesia, macrophage recruitment, and endoneurial TNF-alpha expression. Pain 1998;74:3542.

30. Cui JG, Holmin S, Mathiesen T, Meyerson BA, Linderoth B. Possible role of inflammatory mediators in tactile hypersensitivity in rat models of mononeuropathy. Pain 2000;88:239-48.

31. Shubayev VI, Myers RR. Upregulation and interaction of TNF alpha and gelatinases A and B in painful peripheral nerve injury. Brain Res 2000;855:83-9.

32. Pathak NN, Balaganur V, Lingaraju MC, More AS, Kant V, Kumar D, et al. Antihyperalgesic and anti-inflammatory effects of atorvastatin in chronic constriction injury-induced neuropathic pain in rats. Inflammation 2013;36:1468-78.

33. Erdine S, Yucel A, Cimen A, Aydin S, Sav A, Bilir A. Effects of pulsed versus conventional radiofrequency current on rabbit dorsal root ganglion morphology. Eur J Pain 2005;9:251-6.

34. Tanaka N, Yamaga M, Tateyama S, Uno T, Tsuneyoshi I, Takasaki M. The effect of pulsed radiofrequency current on mechanical allodynia induced with resiniferatoxin in rats. Anesth Analg 2010;111:784-90.

35. Cosman ER Jr, Cosman ER Sr. Electric and thermal field effects in tissue around radiofrequency electrodes. Pain Med 2005;6:405-24.

36. Abejon D, Reig E. Is pulsed radiofrequency a neuromodulation technique? Neuromodulation 2003;6:1-3. 37. Munglani R. The longer term effect of pulsed radiofre- 
quency for neuropathic pain. Pain 1999;80:437-9.

38. Aksu R, Ugur F, Bicer C, Menku A, Guler G, Madenoglu $\mathrm{H}$, et al. The efficiency of pulsed radiofrequency application on L5 and 16 dorsal roots in rabbits developing neuropathic pain. Reg Anesth Pain Med 2010;35: 11-5.

39. Vallejo R, Tilley DM, Williams J, Labak S, Aliaga L,
Benyamin RM. Pulsed radiofrequency modulates pain regulatory gene expression along the nociceptive pathway. Pain Physician 2013;16:E601-13.

40. Ozsoylar O, Akçali D, Cizmeci P, Babacan A, Cahana A, Bolay $\mathrm{H}$. Percutaneous pulsed radiofrequency reduces mechanical allodynia in a neuropathic pain model. Anesth Analg 2008;107:1406-11. 